\title{
Organizations, Plans, and Schedules: An Interdisciplinary Perspective on Coordinating AI Agents
}

\author{
EDMUND H. DURFEE ${ }^{1}$
}

Dept. of Electrical Engineering and Computer Science

University of Michigan, Ann Arbor, MI 48109, USA

\section{CONTENTS}

Synopsis

158

1. Introduction ................................................................................ 158

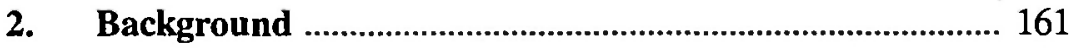

2.1 Organizations ………………………………………...... 161

2.2 Plans ............................................................................ 163

2.3 Schedules ........................................................................ 164

2.4 Other Metaphors …………………….......................... 164

3. Partial Global Planning .......................................................... 165

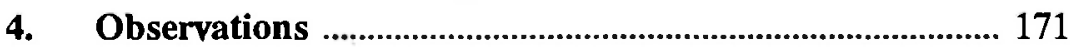

4.1 Abstracting Activities .................................................... 171

4.2 Organizational Self-Design ........................................ 173

5. A Common Representation for Coordination ……............. 175

6. Conclusions and Current Directions ................................... 180

Acknowledgements ............................................................................ 182

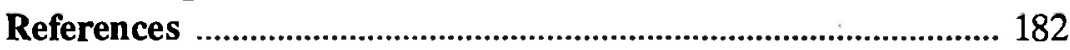

1 This research has been sponsored, in part, by the National Science Foundation under grant IRI-9010645, under coordination Theory and Collaboration Technology grant IRI-9015423, and under a Presidential Young Investigator Award IRI-9158473. Partial support has also come from the University of Michigan Rackham Graduate School. 


\section{SYNOPSIS}

Artificial intelligence, on the one hand, and behavioral and social science on the other, are inextricably related because the ability to coordinate in multiagent environments is fundamental to intelligence. Distributed artificial intelligence (DAI) has used several different social metaphors as the basis for building computational theories of intelligent coordination, with significant success. However, the real challenge in developing general theories of coordination is in bridging between the different perspectives implicit in the various social metaphors. In this paper, I describe a line of research that has led to a view of coordination as a distributed search problem, where the search space provides a common representation for organizations, plans, and schedules. The evolving approach is thus a candidate foundation for a truly interdisciplinary study of coordination from both computational and social perspectives.

\section{KEYWORDS}

Cooperative Problem Solving, Distributed AI, Organizations, Distributed Computing, Planning, Negotiation.

\section{INTRODUCTION}

Most Artificial Intelligence (AI) research focuses on developing intelligent agents that are designed to act by themselves in their environments. The research I describe here challenges that design decision. Difficult tasks in realistic environments generally require an intelligent agent to interact with other intelligent agents in order to cooperatively achieve goals, share information, avoid conflicts, resolve conflicts 
when they arise, compete successfully for resources, and so on. Yet, the computational means for representing this type of "social" knowledge and for using the knowledge to coordinate decisions are still poorly understood and difficult to implement in any practical manner. A fundamental goal of the subfield of AI called Distributed AI (DAI) is to develop the computational theories and mechanisms for identifying, representing, and manipulating knowledge about intelligent coordination, so that $\mathrm{AI}$ agents can be individually and collectively intelligent.

Coordination has been extensively studied by researchers in diverse social sciences such as organization theory, anthropology, operations research, and sociology. These researchers have employed the tools and methodologies of their fields to increase our understanding of the nature of intelligent coordination. The rich heritage of concepts and ideas from these fields have greatly impacted DAI since its inception. One of my objectives in this paper is briefly to review DAI research that has drawn on diverse social metaphors /5/ to highlight the strong interdisciplinary nature of the field.

However, the diverse perspectives of the different social sciences have led to viewing common issues of coordination differently. For example, coordination generally requires a meshing of behaviors to promote cooperation and avoid conflict, but this process of restructuring how agents interact has been described alternatively as organizing, as group planning, and as scheduling. As a consequence, DAI researchers have at times found it difficult to directly compare alternative approaches that are based on different metaphors, even though the overall goals of the approaches are the same.

My second objective in this paper is to describe a line of research that has tried to distill fundamental and shared issues, concepts, and 
techniques out of the different metaphors by reducing them to common computational terms. In particular, my goal is to motivate a knowledgebased, computational theory of coordination that incorporates concepts from organizational theory, AI planning, and scheduling science. The powerful idea behind this integrated theory is that a single representation space can accommodate reasoning about organizations, plans, and schedules. As a consequence, the approach I describe not only draws together previously distinct DAI techniques, but also might help the relevant social sciences to draw new parallels between themselves.

Studying coordination from an AI perspective means developing, testing, and revising computational theories and mechanisms, and in this paper I describe a line of research based on this iterative methodology. Because I am trying to provide a high-level introduction to the issues and conceptual contributions of the research, I do not delve into technical details about data structures and algorithms; the interested reader is encouraged to read the works I cite for more information. I should also make clear that my line of research is directed toward developing AI agents that can coordinate well enough to meet the needs of their environment; getting them to coordinate in exactly the way that people do is not a primary goal.

I begin the remainder of this paper by describing separate DAI approaches to coordination based on insights from organizational theory, from AI planning, and from scheduling science (Section 2). Based on the strengths and limitations of these separate approaches, I have pursued a line of research that has brought together a number of these ideas into a single framework called Partial Global Planning (Section 3). This framework has served to overcome weaknesses of prior approaches by melding them together, and yet in evaluating partial global planning I observed problems that still could not be easily 
resolved in such a hybrid approach; what is called for is an approach that treats organizing, planning, and scheduling identically (Section 5). This hypothesis forms the core of my ongoing work in forming a new computational theory of coordination that views the process of coordination, whether at an organizational, planning, or scheduling level, as a search through an hierarchical space of individual and collective behaviors. I summarize this line of research, outline current research directions, and speculate on its potential implications for the social sciences in Section 6.

\section{BACKGROUND}

Many AI paradigms have been based more-or-less loosely on human problem-solving metaphors. Correspondingly, many DAI paradigms have at their roots insights from how communities of people (or other natural agents) collectively solve problems. Three prevalent social metaphors are organizations, team plans, and collective scheduling. In the following, I briefly summarize DAI approaches that have drawn on these three different metaphors, and conclude with an overview of other social metaphors that have also played a role in DAI. More complete surveys of many of these approaches can be found elsewhere $/ 3,11,16,28 /$.

\subsection{Organizations}

Because each of us is involved in a variety of human organizations, a familiar and powerful metaphor in DAI has been to draw on formal and informal notions of organizations $/ 19,27 /$. From an organizational perspective, each AI agent in a group plays a particular organizational 
role; if these roles are defined and assigned appropriately, the group as a whole can work as a coherent team.

A simple example of using an organization can be found in the work of Gasser /20/, which considers the "pursuit problem" where four "predators" must pursue and surround a "prey" on a grid. The predators identify four organizational roles, each corresponding to occupying a location (or region) on a different side of the prey. By assigning these roles among themselves appropriately, they can work in concert to surround and capture the prey. The challenge is for the agents to settle questions about what roles should be filled and who should fill them, and Gasser thus views the formation of organizations as based on "the settled and unsettled problems about belief and action through which agents view other agents". Other work on the pursuit problem /2,35/ has focussed on alternative organizations among predators for assigning responsibilities among themselves.

More complicated task domains require organizations that assign more abstract roles to agents so that they have sufficient flexibility to adapt to changing task demands. However, an agent working within such an organization needs sophisticated local decisionmaking capabilities in order to determine which of its possible activities it should perform to enhance the collective effort most. The coupling of organizational structures and sophisticated local control has been studied by Corkill and Lesser $/ 8,10$, who provide agents with jointly-known organizational structures that specify a general set of long-term responsibilities and interaction patterns between them. The organization helps the agents generate and selectively share information that is of importance to the group. At the same time, the agents are not overly constrained by the general organizational structures, so that each can remain reactive and opportunistic as the situation it faces evolves. 
A specific organizational structure is suited to certain demands on coordination, and could be ineffective given other demands. An ongoing challenge has been to characterize the attributes of situations and organizations so as to be able to prescribe an organizational structure based on the classification of the current situation /30/. Given such knowledge, agents could redesign their organization to meet the needs of new situations $/ 25 /$.

\subsection{Plans}

Planning has been a primary concern of AI for many years, so it is not surprising that many $\mathrm{AI}$ researchers have viewed the problem of coordination as a problem of planning for multiple AI agents. This perspective has resulted in techniques that have inherented both the strengths and weaknesses of planning paradigms. In fairly predictable application domains, AI planning paradigms that search among different orderings of plan steps provide a powerful means for ensuring that all goals will be achieved. Such assurances are important in many multiagent domains as well. For example, in air-traffic control, fully predicting and resolving interactions (collisions) before taking action is better than "flying by the seat of your pants". However, because plans are generally viewed as programs to be executed /1/, they can lock agents into a course of action that, as things evolve, is inopportune. Moreover, foreseeing and planning around all possible interactions can be computationally costly and can place great demands on communication in a distributed system.

AI research in planning for multiple agents can be classified into two main approaches. The first is centralized multiagent planning, in which one of the agents, chosen either by the other agents $/ 4$ or by the 

disciplinary Perspective on Coordinating AI Agents human designer $/ 21 /$, collects the separate plans of the agents and analyzes them to find potential conflicts. It resolves conflicts by suggesting alterations to plans or by inserting additional synchronization steps. The second main approach is distributed multiagent planning $19,22,26 /$, where agents model each other as they develop their plans and exchange information about how each will affect the world in order to identify and resolve conflicts.

\subsection{Schedules}

AI research in scheduling has predominantly focussed on manufacturing concerns, where the proper scheduling of equipment and manpower impacts the throughput and efficiency of a factory. Because scheduling often requires coordination of many resources that are physically or functionally distinct, researchers at Carnegie-Mellon have investigated a distributed approach to scheduling $/ 36 /$. The theme underlying this work on scheduling is that constraints on how resources are applied must be generated and propagated to generate suitable schedules. As a consequence, DAI approaches to scheduling generally resemble constraint satisfaction techniques that have been modified to allow knowledge of and communication about constraints to be distributed.

Distributed constraint satisfaction is similarly at the core of the multistage negotiation protocol $/ 7 /$. The goal of this protocol is to schedule the allocation of communication network resources so as to establish as many of the desired end-to-end connections as possible.

\subsection{Other Metaphors}

DAI researchers have exploited other social and natural metaphors 
as well. Economics, for example, has provided a rich set of ideas for DAI, including decision-theoretic and game-theoretic techniques for modeling multiagent decisionmaking in cooperative and competitive situations $32,38,39 \%$. A particularly useful metaphor has been viewing the decomposition and allocation of tasks among $\mathrm{AI}$ agents as a form of contracting. Contracting, as first embodied in the Contract-Net protocol $134 /$, has been a fundamental paradigm in the field, especially for problems requiring tasks to be shared among agents $/ 33 /$. Finally, metaphors from ecological systems /24/ and human discourse /6,23/ have also impacted the field.

\section{Partial global PlanNing}

Partial global planning /17,13/ capitalizes on the strengths of the organizational, planning, and contracting approaches by uniting them into a single, hybrid framework. The goal of this framework is to get multiagent planning's benefits of detailed, situation-specific coordination without its excessive computation and communication costs, which is accomplished because the jointly-known organizational structures effectively prune the space of possible plans to keep the problem tractable. In addition, partial global planning views contracts as jointly held plans that specify future exchanges of tasks and results among agents. Hence, within partial global planning, coordination involves both sharing tasks and sharing results, both adhering to long-term organizational roles and reactively planning to achieve short-term objectives.

Partial global planning has been designed to coordinate agents that are cooperatively solving problems $/ 16 /$. During cooperative distributed problem solving, agents solving subproblems of the overall problem must share their results to converge on overall solutions. For example, partial global planning has been implemented and tested in the context of 
a distributed sensor network application, as simulated in the Distributed Vehicle Monitoring Testbed (DVMT) /28/. Fig. 1 shows a muchstudied example problem in the DVMT, in which a vehicle moves among four sensors, each of which is attached to a separate agent (sensor_ $i$ is connected to agent_i) that must track vehicles within its own sensed range and share tracked information with other agents to build a map of vehicle movements for the entire area.

The agents should coordinate their problem-solving activities in order to work as an effective team. Once all the data have arrived, coordination among them should involve:

- assigning tasks to agent_4 so that its resources are used even though it does not receive any data to process from its own sensor;

- getting agent_1 to focus on processing data for the long vehicle track first (even though the data in its upper left corner is more strongly sensed), because the agents have greater confidence in the correctness of longer tracks;

- having agents 1,2 , and 3 assign data in their overlapping areas among themselves so as to avoid redundant processing activity;

- having agent_1 send agent_2 a partial track adjacent to agent_2's region early on, so that agent_2 can filter out much of its noisy data.

In partial global planning, an agent represents its anticipated future problem-solving activities as a problem-solving plan. In vehicle monitoring, for example, a problem-solving plan would indicate what data the agent expects to be processing when, and what results it anticipates forming as a result. To gain the benefits of coordination listed above, useful results must be formed and shared in a timely manner, so the 
agents must coordinate their individual plans to work as an effective team. They do this by exchanging information about the major plan steps they will be taking and the results they are expecting. As an agent receives this information from others, it determines whether it has any local plans to pursue related results, and if so it combines the received information with a summary of its relevant local plan(s) into a larger, more global plan. Because this representation can change over time as information arrives, and might not reflect the activities of every agent in the network, it is called a partial global plan.

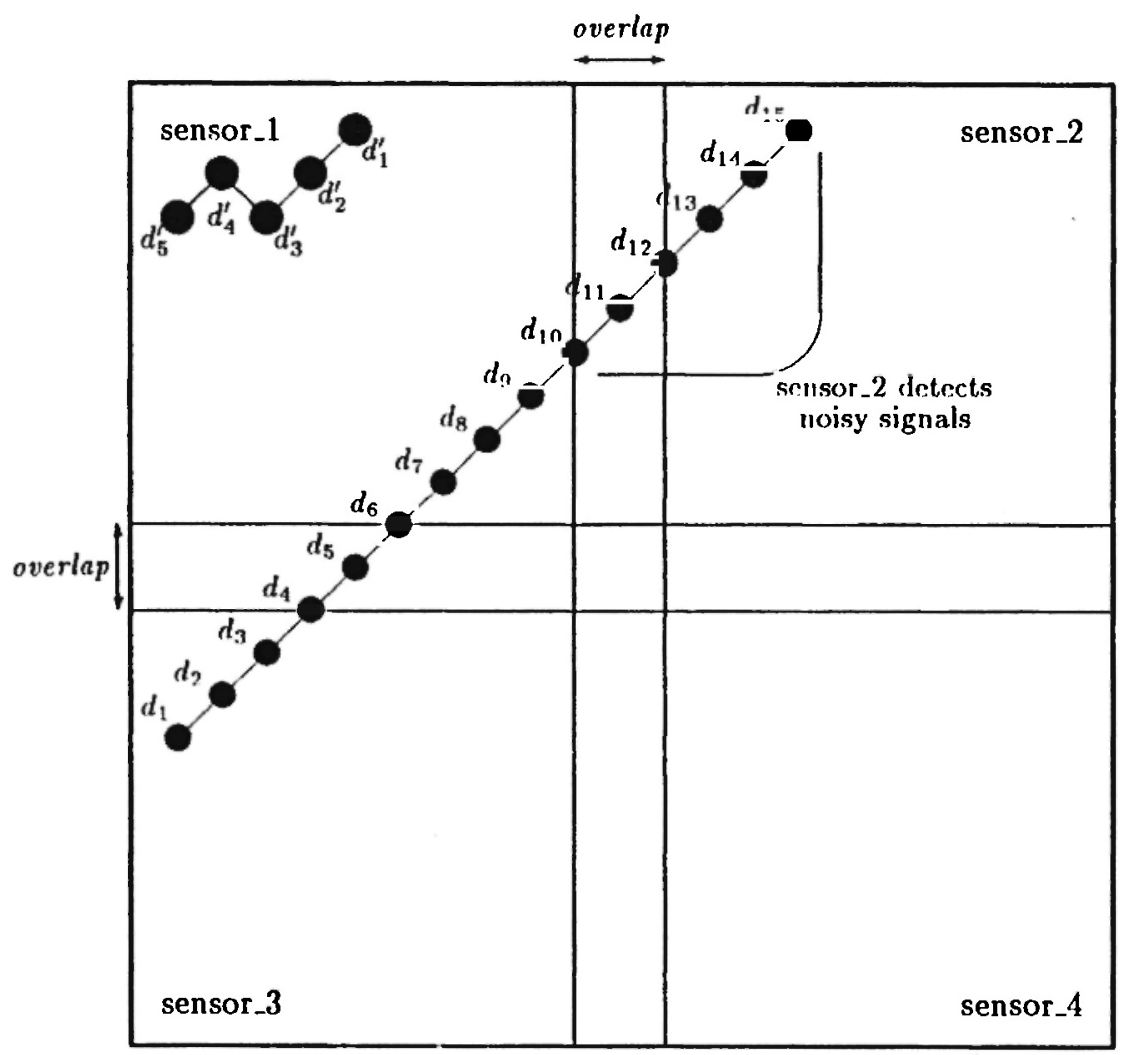

Fig. 1: A Distributed Vehicle Monitoring Example. 
In a partial global plan, an agent has a representation of the interleaved major plan steps of the participating agents. From this view, it can hypothesize better orderings and allocations of steps to agents, which can then be used to modify its own local plans accordingly. For example, agents 1 through 3 in Fig. 1 exchange summaries of their local plans and each seaprately builds a partial global plan corresponding to the goal of forming a vehicle track spanning their combined areas. Each can use its version of the partial global plan to identify better orderings of its local activities, such as when agent_l changes its plan so as to generate and send a partial track to agent_ 2 to help it filter out its noisy data sooner.

Agents initiate partial global planning by voluntarily exchanging information about their most highly rated plans, and they also send updated information whenever their local plans have changed substantially. Because agents are independently and asynchronously forming, revising, and using partial global plans, they might never actually converge on identical views of coordination due to communication delays and domain dynamics. Partial global planning still helps them to coordinate satisfactorily even in these cases, and gives them enough local autonomy to respond to important local changes in their information $/ 12 \%$. An extensive discussion of how agents use partial global planning to coordinate successfully in the scenario of Fig. 1 can be found elsewhere $/ 13 /$.

If the exchange of plan information among agents is uncontrolled, the agents could swamp the communication channels and each other with unnecessary information. Partial global planning views coordination as a distributed problem to solve in its own right, and employs an organizational approach to control the exchange of plan information. Besides having a domain-level organizational structure that specifies the 
agents' roles in solving their application problem, the agents also have common knowledge about the meta-level organization that specifies their roles in solving coordination problems. Thus, agents are participating in multiple organizations at the same time.

The ability of agents to participate concurrently in multiple organizations allows interesting parallels to human societies and enterprises. The meta-level organization essentially dictates a management control structure in which some agents' principal contributions are in coordinating the ongoing activities of others. Meanwhile, the domain-level organization controls the flow of results within the enterprise. While the meta-level and domain-level organizations generally have a high degree of correspondence (as in a professor who integrates the results and directs the research of students), they can also at times be decoupled (as in a facilities manager who ensures that resources are maintained and utilized without necessarily knowing about what the resources are producing or why).

That a proper choice of organizational structures can strongly impact the overhead and quality of coordination in an enterprise is commonly known among organizational theorists, and experiments with partial global planning provide additional verification for this intuition. In particular, as the number of agents increases, choices of organizational structures strongly impact the combinatorics of coordination. I have experimented with networks of 10 sensors and 10 or 11 agents. The details of these experiments are given elsewhere /17/; here I summarize some of the findings.

When I employ 10 agents, they use a domain-level organization that is lateral (any agent can combine results from others), while in an 11 agent network, the 10 agents connected to sensors form local track 
Organizations, Plans, and Schedules: An Interdisciplinary Perspective on Coordinating Al Agents hypotheses which they send to the eleventh agent who integrates the hypotheses. For meta-level organizations, I initially considered both lateral and centralized organizations. I quickly discovered that the overhead of a lateral organization is extremely high, since each agent is collecting and reasoning about the plans of all agents in order to coordinate its local plan. However, regardless of the domain-level organization, the lateral meta-level organization allows agents to be locally more responsive to unexpected situations because they can unilaterally change their plans without approval of a higher authority. A centralized meta-level organization incurs much less overhead because only one agent is reasoning about collective activity, but with many agents to coordinate the central manager can quickly become a bottleneck and a reliability hazard.

In cases where central management is needed but is beyond the capabilities of a single agent, human organizations often adopt a hierarchical organization. Using a hierarchical meta-level organization, the simulation realized many of the intuitive phenomena that might be expected. For example, the computational overhead in a hierarchical organization is reduced because middle managers are coordinating fewer agents (in these experiments, the 2 middle managers coordinate 5 agents each), and then are sending the coordinated 5-agent partial global plans to the single top manager who only has to iron out wrinkles between these plans. In the representation, the top manager still has the details of all 10 agents' activities, but because much of the coordination has been instituted by the middle managers, the top manager's search for better coordination is significantly simplified. The cost of this reduction of overhead is, as might be expected, slower responsiveness in the individual agents (since they must wait for permission to change plans to be passed through more levels of bureaucracy) and more communication (within the bureaucracy). 


\section{OBSERVATIONS}

As I have outlined, partial global planning has several advantages over its predecessors, including the ability to constrain the generation of plans within organizational bounds, to coordinate plans to improve collective performance, and to institute altemative organizations at both the domain and meta levels to balance competing needs including reducing communication, reducing computation, increasing reliability, and increasing responsiveness to unexpected events. Yet, partial global planning also has major limitations, two of which are:

Scale-up: While a hierarchical organization alleviates certain scale-up difficulties, the way that partial global planning represents collective activities as the union of individual activities makes representing and reasoning about a large number of agents unwieldy.

Organizational self-design: While partial global planning has been developed such that organizational structures are explicit and modifiable, decisions about what organization to institute for a given scenario are left to the human experimenter rather than to the agents themselves.

I now turn to issues in overcoming these limitations.

\subsection{Abstracting Activities}

Higher levels of management need to simplify the representation of organizational sub-units. For example, a corporate president might view the corporation as being composed of a small number of entities 
(divisions for production, design, purchasing, and so on). While each entity is itself a complicated multiagent system, the corporate president can typically perform his or her managerial duties without knowing the internal structure of the divisions. That is, the corporate preside coordinate the divisions based on abstractions of them, but only if those abstractions are appropriate. For example, an abstraction indicating how many people are in each division and what their average pay is would be useful when deciding on budget cuts, but could be useless when it comes to shepherding a new product through the pipeline.

Complications in abstracting collective activity crop up within partial global planning as well. In a 10-agent, hierarchical organization, for example, how should each middle manager abstract the activities of the 5 agents it is responsible for such that the abstraction will be of use to the top manager? If the two 5-agent teams are developing pieces of the same track, then each team should be abstracted to highlight what partial solutions it is forming at different times so the top manager can coordinate the exchange of partial solutions that help the teams form compatible pieces. Alternatively, the teams might be working on independent solutions, but they might each be planning on using a solution method that involves a common, non-sharable resource (such as an agent that is streamlined for integrating partial results). For the top manager to coordinate the teams' access to shared resources efficiently, it needs abstractions that emphasize how the teams are going to be achieving their results rather than what results they expect to produce.

Thus, partial global planning led me to observe that teams of problem-solving agents should sometimes emphasize what subproblem solutions they are forming, sometimes emphasize how they are forming solutions, and sometimes emphasize when they are solving particular subproblems. There are times when who is needed on a team is of 
primary importance, and other times when where information or processing responsibility reside is critical. Moreover, properly abstracting collective activities might require some amount of exploration, as agents revise their abstractions to emphasize different attributes based on the quality and complexity of coordination given the current choice of attributes. In short, then, while using abstractions of collective behavior to reduce communication and computation in hierarchical organizations is an intuitive solution, flexibly constructing the right abstractions is far from easy.

\subsection{Organizational Self-Design}

While partial global planning supports dynamic coordination of problem solving plans, the agents have no facilities for changing their relatively static organizational structure. As a result, an agent that is chronically overburdened and another one that is habitually underutilized will repreatedly use partial global plans to relocate particular tasks from one to the other. While such repeated coordination is better than none at all, the chronic imbalance of their loads should instead be resolved once and for all by revising their common organizational structure so that a portion of the tasks that go to the overworked agent become the responsibility of the underworked agent. Fixing the organization once in this case is much more cost effective than continually repairing load imbalances due to a faulty organization.

An impediment to organizational self-design in partial global planning is the distinction made between plans, which are dynamically generated, exchanged, and modified, and organizations, which are static and assumed commonly known. One way of viewing the partial global planning approach is as a means for agents who have broadly-defined organizational roles to develop and exchange information that narrows 
down the role each is playing in the current situation. From this perspective, an organization really is unmodifiable.

But this really is an artifact of the hybrid approach taken in partial global planning, where multiagent planning and organizational structuring remain distinct despite being united in one framework. To truly allow organizational self-design, the agents should be able to generate, exchange, and modify their organizational roles as they can their plans. In other words, plans and organizations should be treated identically.

Toward this end, note that, as a collection of interacting agents abstracts its activities, details become lost. For example, specifics about what partial results will be formed and when can be blurred, leading to a view of the agents as generating a variety of results over an extended time period. As the time period gets longer, in fact, the representation of collective activity looks less like a plan, and more like an organization! Individuals (or the entire team) become associated with a general set of expectations, which constitutes an organizational role. Characterizations of results to be produced over an extended time period represent what other agents (or collections of agents) can expect from the team, without details of how the team accomplishes its results.

This gradual transition from plans to organizations as a consequence of abstraction can also proceed in the other direction. An organization with its associated personnel, processes, and products, can be decomposed into suborganizations. The issues involved in choosing appropriate attributes for decomposition -- to emphasize some aspects of collective activity over others - are analogous to choosing appropriate attributes to emphasize when abstracting collective plans. Thus, decomposing an organization into a product hierarchy is analogous to reducing abstraction (specializing) along the attribute of what results different 
subgroups will be achieving. On the other hand, decomposing an organization into a functional hierarchy maps to specializing along the attribute of how subgroups are acting (the expertise and resources they are applying to their tasks). Organizations can also be decomposed to emphasize spatial relationships (such as coordinating a distribution of franchises, each of which has identical functional and product capabilities), and temporal relationships (such as transitioning to new products or functions in response to changing market demands), corresponding to the where and when attributes we identified in partial global planning.

As specialization along different dimensions proceeds, the representation of activity begins to resemble not so much an organization, or even a plan, but instead a schedule. By nailing down precisely when each activity will take place, along with the processes, resources, agents, and expected results of each activity, we can transform our representation into a detailed schedule.

\section{A COMMON REPRESENTATION FOR COORDINATION}

Based on these observations, I hypothesize that plans in the AI sense, organizations in the management sense, and even schedules in the operations research sense, are in fact manifestations of the same type of entity. I call this hypothesis:

The Common Representation for Coordination Hypothesis: Organizations, plans, and schedules have a common representation, but differ in their degree of specificity along different descriptive dimensions. 
In our ongoing work, we call this common representation a behavior. The descriptive dimensions for a behavior, as gleaned from experiences with partial global planning, include what the behavior is trying to achieve (the goals), how it is trying to achieve the result (the methods), who is participating in the behavicr (the participants), when the behavior is active (the time intervals), where the behavioral activities are taking place (the regions), and why the behavior has been instituted (the motivations for pursuing the goals). While this set of dimensions is only preliminary, it has proven a useful starting point for representing and reasoning about alternative forms of coordination.

These dimensions form the "slots" for the behavioral representation. Instances of behaviors then are combined in a linked network, where a behavior is linked to its more abstract and more specialized behavioral instances. As a simple example, consider the partial hierarchy of behaviors shown in Fig. 2. At the most abstract, long-term level, the behavior represents an organizational objective: The (fictitious) Computer Corporation wants to increase its US computer sales in the 1990 s by creating more demand for its product over that interval of time. Tracing downward in the hierarchy, the current plan for achieving the organizational objective that the New York subsidiary has adopted is to first increase the number of users of the product by giving computers away, and then using testimonials from satisfied users to convince additional buyers. Tracing further downward, toward the end of giving computers away, the subsidiary has scheduled sweepstakes.

The behavior hierarchy thus represents the composition and decomposition of an enterprise in terms of time, space, goals, means, personnel, and motivation. Different parts of an enterprise will have different pieces of this hierarchy, and communication between suborganizations should use the proper behavioral representation. For example, if the New York and the Boston subsidiaries of Computer Corporation want to avoid competing with each other, they might begin 


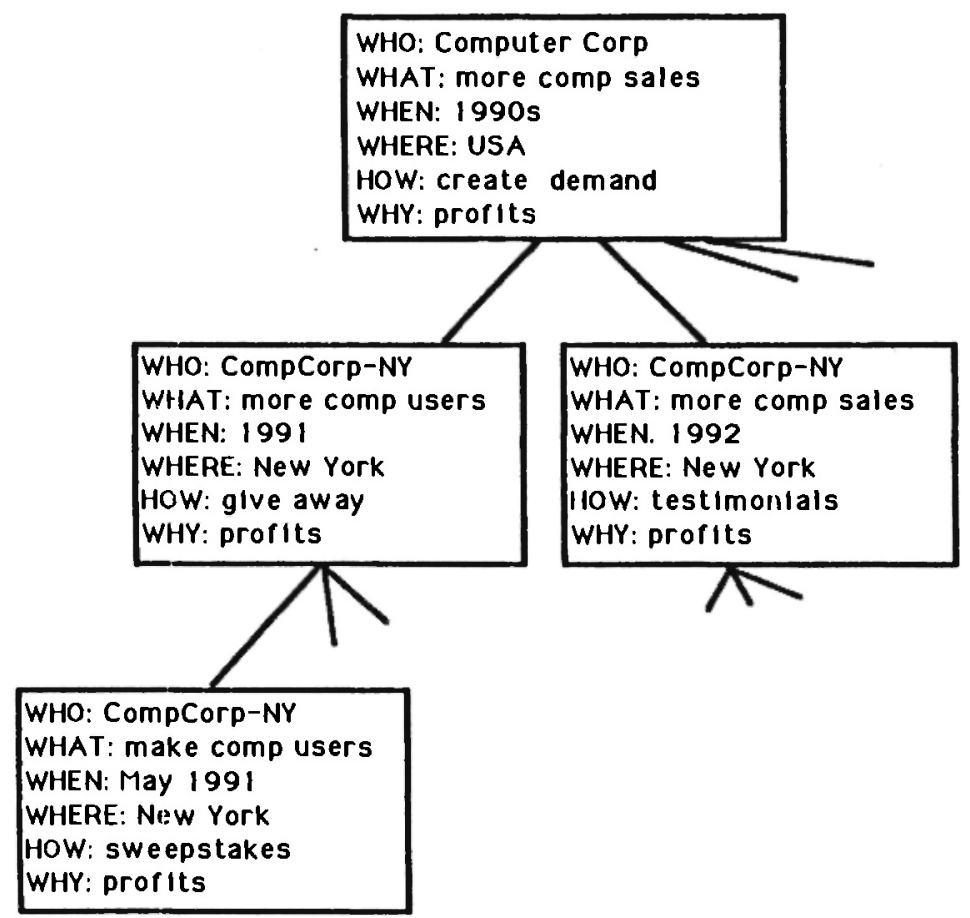

Fig. 2: Partial Hierarchy for Computer Corporation.

by communicating about their upcoming behaviors at a fairly abstract level to detect potential competition, and then communicate further details only when competition seems likely /14/. Thus, if the Boston subsidiary is increasing demand through rebates, the subsidiaries should further investigate how the combination of sweepstakes and rebates will impact their overlapping sales areas.

The behavior hierarchy hence represents a search space in which agents (or groups of agents) attempt to find coordinated joint behaviors. It is important to note that the search space is not static. Consider, for example, what happens when a second corporation, called Department 


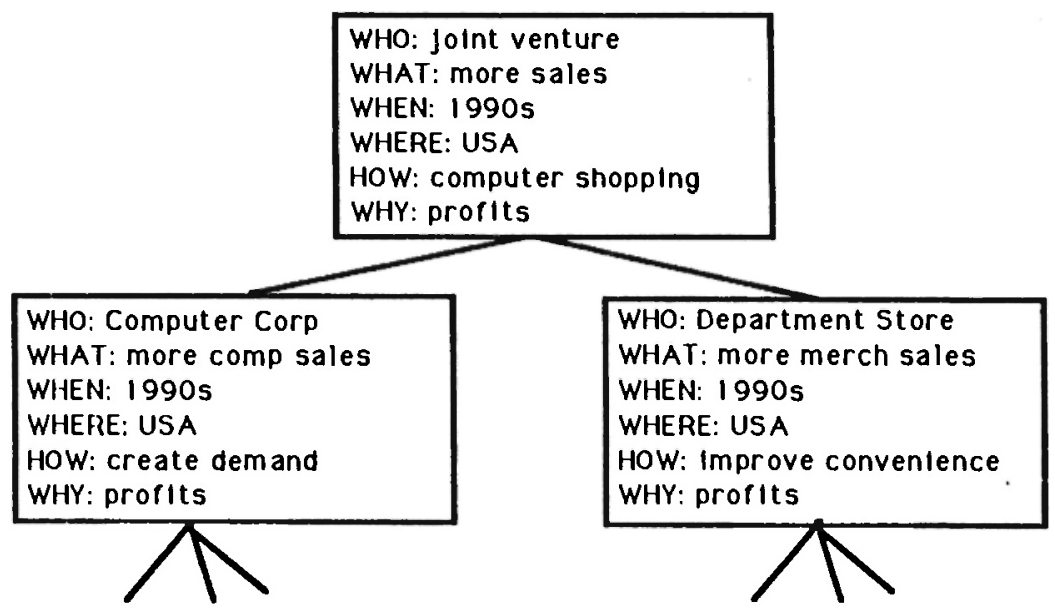

Fig. 3: Generating a Joint Behavior.

Store, shares the environment with Computer Corporation (Fig. 3). The Department Store wants to sell more merchandise in the US during the 1990 s by making shopping more convenient. If the two companies communicate about their organizational objectives and search for potential joint activities, they could generate a behavior corresponding to a joint venture. In this joint venture, both companies achieve their separate objectives through instituting a service by which customers can shop from home using computers.

The process of coordination, therefore, reduces to a search through the space of possible joint behaviors. This applies equally to whether the coordination is occurring at the organizational, planning, or scheduling level. Because the hierarchical behavior space is distributed among the different agents (agents typically have limited knowledge about others' behaviors), coordination amounts to a distributed search.

We are working toward fleshing out and formalizing a unified 
theory of coordination in which the process of coordination is a knowledge-based search through a hierarchical behavior space. To date, we have identified five components of this theory:

Hierarchical Behavior Space: The linked data structures that make it possible to represent organizations, plans, and schedules within a single search space.

Distributed Search Protocol: The algorithms and conventions that agents use when communicating about behaviors during the search for more coordinated group behaviors.

Local Search Algorithms: The algorithms that an agent can use to generate alternative behaviors when searching for more coordinated group behaviors.

Metrics: The criteria for determining whether one combination of behaviors is superior to another, based on individual or group performance characteristics.

Control Knowledge and Heuristics: The knowledge (often heuristic) that focuses search for coordinated behavior into promising areas of the space.

Further details about this evolving theory are given elsewhere /15/, and are beyond the scope of this article. To date, our current work has been on elaborating these ideas within several application domains, most notably a simulated robotic domain in which robots must coordinate deliveries of articles from producers to consumers. 


\section{CONCLUSIONS AND CURRENT DIRECTIONS}

In this paper, I have traced a line of research in the field of distributed AI that has drawn on, and hopes to contribute to, the social sciences. Just as traditional AI serves as a melting pot for ideas from such diverse fields as psychology, linguistics, and neurobiology, distributed AI brings togehter ideas from fields like sociology, organization theory, anthropology, and discourse analysis. The rich substrate of ideas from these fields has provided fertile ground on which DAI can grow. In turn, DAI's unique perspective on viewing intelligence in terms of knowledge and search spaces holds the promise of shedding new light on concepts and techniques in the social sciences. While the impact on the social sciences of the evolving theory of coordination as distributed search remains to be seen, the more important conclusion is that computing and artificial intelligence are indeed fundamentally intertwined with the social and behavioral sciences, and that building on these connections can enrich all of the fields.

As I have outlined, insights from the social sciences have contributed and will continue to contribute to our work. Considering the five components of the evolving theory I just described, for example, opportunities to exploit the powerful ideas from the social sciences include the following:

Social sciences have developed extensive knowledge, much of it heuristic, for guiding the search for appropriate group behaviors. For example, organizational theorists have developed guidelines for searching among alternative organizational structures to match a structure to the collective effort at hand. Vroom $/ 37 /$ has developed a decision tree for choosing among organizational alternatives. Decision trees are familiar in AI, and the challenge is to map questions 
asked in traversing the tree (such as "Is the problem structured?") into well-defined metrics for $\mathrm{AI}$ agents.

- Sciences such as operations research have developed a variety of search algorithms, such as different scheduling algorithms, for generating alternative ways to coordinate.

- Numerous social sciences, ranging from management science and operations research to sociology and anthropology have identified various metrics for evaluating group behavior, including productivity, reliability, and adaptability.

- Similarly, communication protocols have been studied in diverse settings, ranging from electronically-mediated message passing in highly-structured organizations to conversation analyses of informal dialogues.

As $\mathrm{AI}$ agents are increasingly introduced into everyday, multiagent settings, the fundamental importance of social knowledge and capabilities become increasingly apparent. For example, research in coordinating AI agents is inextricably tied to research in time-bounded reasoning and acting /18/. Why? Because a large proportion of the "deadlines" imposed on real-time systems, including real-time AI systems, are due to interagent commitments rather than to inherent environmental demands. An intelligent agent working under time limitations should not only be able to adapt its behavior to attempt to meet deadlines, but should also be able to reason about the deadlines themselves and at times handle deadlines by negotiating extensions.

In conclusion, then, DAI has borrowed ideas from the social sciences in order to make inroads into building AI agents that can 
achieve their goals in multiagent worlds, but many more insights remain to be used. At the same time, the methodologies and objectives of DAI can lead to new outlooks on social theories, such as the work I have described in this article that develops a unified view of coordination as distributed search. If we are fortunate, then the young science of artificial intelligence, which initially only borrowed ideas from the older social sciences, is ready to contribute useful insights back to the social sciences, so that in time a truly interdisciplinary "coordination science" $B 1 /$ will unite the relevant fields.

\section{ACKNOWLEDGEMENTS}

Victor Lesser has provided important feedback and insights in this work, and was instrumental in developing the partial global planning framework. Thomas Montgomery has helped further the more recent aspects of this research into developing the theory of coordination based on a distributed search paradigm. Also, discussions with other members of the University of Michigan Distributed Intelligent Agents Group (the "Michigan DIAG") have helped in formulating the ideas within this paper.

\section{REFERENCES}

1. Agre, P.E. and Chapman, D., What are plans for? Technical Report AI Memo 1050, Massachusetts Institute of Technology, September 1988.

2. Benda, M., Jagannathan, V. and Dodhiawalla, R., On optimal cooperation of knowledge sources, Technical Report BCS-G201028, Boeing AI Center, Boeing Computer Services, Bellevue, WA, August 1985. 
3. Bond, A.H. and Gasser, L., Readings in Distributed Artificial Intelligence, Morgan Kaufmann Publishers, San Mateo, CA, 1988.

4. Cammarata, S., McArthur, D. and Steeb, R., Strategies of cooperation in distributed problem solving, in: Proceedings of the Eighth International Joint Conference on Artificial Intelligence, Karlsruhe, Germany, p. 767-770, August 1983. (Also published in Readings in Distributed Artificial Intelligence, Bond, A.H. and Gasser, L., Eds., Morgan Kaufmann, p. 102-105, 1988.)

5. Chandrasekaran, B., Natural and social system metaphors for distributed problem solving: Introduction to the issue, IEEE Transactions on Systems, Man, and Cybernetics, SMC-11(1), 1-5, January 1981.

6. Cohen, P.R. and Levesque, H.J., Rational interaction as the basis for communication, in: Cohen, P.R., Morgan, J. and Pollack, M.E., Eds., Intentions in Communication, MIT Press, 1990.

7. Conry, S.E., Meyer, R.A. and Lesser, V.R., Multistage negotiation in distributed planning, in: Bond, A.H. and Gasser, L., Eds., Readings in Distributed Artificial Intelligence, Morgan Krufmann, p. 367-384, 1988.

8. Corkill, D.D. and Lesser, V.R., The use of meta-level control for coordination in a distributed problem solving network, in: Proceedings of the Eighth International Joint Conference on Artificial Intelligence, Karlsruhe, Germany, p. 748-756, August 1983. (Also appeared in Computer Architectures for Artificial Intelligence Applications, Wah, B.W. and Li, G.-J., Eds., IEEE Computer Society Press, p. 507-515, 1986.)

9. Corkill, D.D., Hierarchical planning in a distributed environment, in: Proceedings of the Sixth International Joint Conference on Artificial Intelligence, Cambridge, Massachusetts, p. 168-175August 1979. (An extended version was published as Technical Report 79-13, Department of Computer and Information Science, 
University of Massachusetts, Amherst, Massachusetts 01003, February 1979.)

10. Corkill, D.D., A Framework for Organizational Self-Design in Distributed Problem Solving Networks, Ph.D. Thesis, University of Massachusetts, February 1983. (Also published as Technical Report 82-33, Department of Computer and Information Science, University of Massachusetts, Amherst, Massachusetts 01003, December 1982.)

11. Decker, K.S., Distributed problem-solving techniques: A survey, IEEE Transactions on Systems, Man, and Cybernetics, 17, 729740, 1987.

12. Durfee, E.H. and Lesser, V.R., Predictability versus responsiveness: Coordinating problem solvers in dynamic domains, in: Proceedings of the National Conference on Artificial Intelligence, p. 66-71, August 1988.

13. Durfee, E.H. and Lesser, V.R., Partial global planning: A coordination framework for distributed hypothesis formation, IEEE Transactions on Systems, Man, and Cybernetics, 21(5), September 1991 (Special Issue on Distributed Sensor Networks).

14. Durfee, E.H. and Montgomery, T.A., A hierarchical protocol for coordinating multiagent behaviors, in: Proceedings of the National Conference on Artificial Intelligence, p. 86-93, July 1990.

15. Durfee, E.H. and Montgomery, T.A., Coordination as distributed search in a hierarchical behavior space, IEEE Transactions on Systems, Man, and Cybernetics, 21(6), December 1991 (Special Issue on Distributed AI).

16. Durfee, E.H., Lesser, V.R. and Corkill, D.D., Cooperative distributed problem solving, in: Barr, A., Cohen, P.R. and Feigenbaum, E.A., Eds., The Handbook of Artificial Intelligence, Vol. IV, Chapter XVII, Addison-Wesley, p. 83-137, 1989.

17. Durfee, E.H., Coordination of Distributed Problem Solvers, 
Kluwer Academic Publishers, 1988.

18. Durfee, E.H., A cooperative approach to planning for real-time control, in: Proceedings of the 1990 DARPA Workshop on Innovative Approaches to Planning, Scheduling, and Control, p. 277-283, November 1990.

19. Fox, M.S., An organizational view of distributed systems, IEEE Transactions on Systems, Man, and Cybernetics, 11(1), 70-80, January 1981. (Also published in Readings in Distributed Artificial Intelligence, Bond, A.H. and Gasser, L., Eds., MorganKaufmann, p. 140-150, 1988.)

20. Gasser, L., Rouquette, N., Hill, R.W. and Lieb, J., Representing and using organizational knowledge in DAI systems, in: Gasser, $L$. and Huhns, M.N., Eds., Distributed Artificial Intelligence, Research Notes in Artificial Intelligence, Vol. 2, Pitman, p. 55-78, 1989.

21. Georgeff, M., Communication and interaction in multi-agent planning, in: Proceedings of the National Conference on Artificial Intelligence, Washington, D.C., p. 125-129, 1983. (Also published in Readings in Distributed Artificial Intelligence, Bond, A.H. and Gasser, L., Eds., Morgan-Kaufmann, p. 200-204, 1988.)

22. Georgeff, M., A theory of action for multi-agent planning, in: Proceedings of the National Conference on Artificial Intelligence, Austin, Texas, p. 121-125, August 1984. (Also published in Readings in Distributed Artificial Intelligence, Bond, A.H. and Gasser, L., Eds., Morgan-Kaufmann, p. 205-209, 1988.)

23. Grosz, B.J. and Signer, C., Plans for discourse, in: Cohen, P.R., Morgan, J. and Pollack, M.E., Eds., Intentions in Communication, MIT Press, 1990.

24. Huberman, B., The Ecology of Computation, Elsevier Science Publishers, North-Holland, Amsterdam, 1988.

25. Ishida, T., Yokoo, M. and Gasser, L., An organizational approach 
to adaptive production systems, in: Proceedings of the National Conference on Artificial Intelligence, p. 52-58, July 1990.

26. Konolige, K., A deductive model of belief, in: Proceedings of the Eighth International Joint Conference on Artificial Intelligence, Karlsruhe, Germany, p. 377-381, August 1983.

27. Kornfeld, W.A. and Hewitt, C.E., The scientific community metaphor, IEEE Transactions on Systems, Man, and Cybernetics, SMC-11(1), 24-33, January 1981. (Also published in Readings in Distributed Artificial Intelligence, Bond, A.H. and Gasser, L., Eds., Morgan-Kaufmann, p. 311-320, 1988.)

28. Lesser, V.R. and Corkill, D.D., The Distributed Vehicle Monitoring Testbed: A tool for investigating distributed problem solving networks, AI Magazine, 4(3), 15-33, Fall 1983. (Also published in Blackboard Systems, Engelmore R.S. and Morgan, A., Eds., Addison-Wesley, p. 353-386, 1988; and in Readings from $A I$ Magazine, Engelmore, R., Ed., Vols. 1-5, p. 69-85, AAAI, Menlo Park, California, 1988.)

29. Lesser, V.R. and Corkill, D.D., Distributed problem solving, in: Encyclopedia of Artificial Intelligence, John Wiley \& Sons, p. 245$251,1987$.

30. Malone, T.W., Modeling coordination in organizations and markets, Management Science, 33(10), 1317-1332, 1987. (Also published in Readings in Distributed Artificial Intelligence, Bond, A.H. and Gasser, L., Eds., Morgan-Kaufmann, p. 151-158, 1988.)

31. Malone, T.W., What is coordination theory?, in: Proceedings of the National Science Foundation Coordination Theory Workshop, February 1988.

32. Rosenschein, J.S. and Genesereth, M.R., Deals among rational agents, in: Proceedings of the Ninth International Joint Conference on Artificial Intelligence, Los Angeles, California, p. 91-99, August 1985. (Also published in Readings in Distributed Artificial 
Intelligence, Bond, A.H. and Gasser, L., Eds., MorganKaufmann, p. 227-234, 1988.)

33. Smith, R.G. and Davis, R., Frameworks for cooperation in distributed problem solving, IEEE Transactions on Systems, Man, and Cybernetics, SMC-11(1), 61-70, January 1981. (Also published in Readings in Distributed Artificial Intelligence, Bond, A.H. and Gasser, L., Eds., Morgan-Kaufmann, p. 61-70, 1988.)

34. Smith, R.G., The contract net protocol: High-level communication and control in a distributed problem solver, IEEE Transactions on Computers, C-29(12), 1104-1113, December 1980.

35. Stephens, L.M. and Merx, M.B., The effect of agent control strategy on the performance of a DAI pursuit problem, in: Proceedings of the 1990 Distributed AI Workshop, October 1990.

36. Sycara, K., Roth, S., Sadeh. N. and Fox, M., An investigation into distributed constraint-directed factory scheduling, in: Proceedings of the Sixth IEEE Conference on Artificial Intelligence Applications, 1990.

37. Vroom, V.H., A normative model of managerial decision making, in: Pugh, D.S., Ed., Organization Theory, Penguin Books, Chapter 16, p. 256-276, 1984.

38. Zlotkin, G. and Rosenschein, J.S., Negotiation and task sharing among autonomous agents in cooperative domains, in: Proceedings of the Eleventh International Joint Conference on Artificial Intelligence, p. 912-917, August 1989.

39. Zlotkin, G. and Rosenschein, J.S., Negotiation and conflict resolution in non-cooperative domains, in: Proceedings of the Eleventh International Joint Conference on Artificial Intelligence, p. 100-105, July 1990. 
